\title{
Effective-energy budget in multiparticle production in nuclear collisions
}

\author{
Aditya Nath Mishra ${ }^{1, a}$, Raghunath Sahoo ${ }^{1, b}$, Edward K. G. Sarkisyan ${ }^{2,3, c}$, Alexander S. Sakharov ${ }^{2,4, d}$ \\ ${ }^{1}$ Discipline of Physics, School of Basic Sciences, Indian Institute of Technology Indore, Indore 452017, India \\ 2 Department of Physics, CERN, 1211 Geneva 23, Switzerland \\ ${ }^{3}$ Department of Physics, The University of Texas at Arlington, Arlington, TX 76019, USA \\ ${ }^{4}$ Department of Physics, Kyungpook National University, Daegu 702-701, Korea
}

Received: 13 May 2014 / Accepted: 22 October 2014 / Published online: 18 November 2014

(C) The Author(s) 2014. This article is published with open access at Springerlink.com

\begin{abstract}
The dependencies of charged particle pseudorapidity density and transverse energy pseudorapidity density at midrapidity on the collision energy and on the number of nucleon participants, or centrality, measured in nucleusnucleus collisions are studied in the energy range spanning a few $\mathrm{GeV}$ to a few $\mathrm{TeV}$ per nucleon. The approach in which the multiparticle production is driven by the dissipating effective energy of participants is introduced. This approach is based on the earlier proposed consideration, combining the constituent quark picture together with Landau relativistic hydrodynamics shown to interrelate the measurements from different types of collisions. Within this picture, the dependence on the number of participants in heavy-ion collisions are found to be well described in terms of the effective energy defined as a centrality-dependent fraction of the collision energy. For both variables under study, the effective-energy approach reveals a similarity in the energy dependence obtained for the most central collisions and centrality data in the entire available energy range. Predictions are made for the investigated dependencies for the forthcoming higher-energy measurements in heavy-ion collisions at the LHC.
\end{abstract}

1. Multiparticle production in high-energy particle and nuclear collisions attracts high interest, as, on the one hand, the observables measured first in high-energy collisions, namely multiplicity and transverse energy, are immediate characteristics of this process and bring important information on the underlying dynamics of strong interactions, while on the other hand, this process still eludes its complete understanding. It is already more than half a century as the multi-

\footnotetext{
a e-mail: Aditya.Nath.Mishra@ cern.ch

b e-mail: Raghunath.Sahoo@cern.ch

c e-mail: sedward@mail.cern.ch

d e-mail: Alexandre.Sakharov@cern.ch
}

plicity of the produced particles are considered to be derived by the collision energy [1-3]. In this picture the energy pumped into the collision zone in the very first stage of the collision defines the volume of the interaction lump of participant patterns. Later on, the approach of "wounded" nucleons, or nucleon participants, has been proposed to describe the multiplicity and particle distributions [4]; for a review, see [5]. In this approach the multiplicity is expected to be proportional to the number of participants. However, it was observed at RHIC and similarly at LHC energies, the concept of wounded nucleons does not describe the measurements where the data found to demonstrate an increase with the number of nucleon participants. The problem has been addressed in the nuclear overlap model using Monte Carlo simulation in the constituent quark framework, and the scaling has been shown to be restored [6-11]. In addition, it was observed that the multiplicity and midrapidity-density distributions are similar in $e^{+} e^{-}$and in the most central (headon) nuclear collisions [12] at the same center-of-mass (c.m.) energy pointing to the universality of multihadron production. However, the expectation to observe this type of universality in hadronic and nuclear collisions at similar c.m. energy per nucleon has not been shown by the data where the measurements in hadron-hadron collisions show significantly lower values compared to those in central heavy-ion collisions [13,14].

To interpret these observations, the energy dissipation approach of constituent quark participants has been proposed in $[15,16]$ by two of the authors of this paper. In this picture, the process of particle production is driven by the amount of energy deposited by interacting participants into the small Lorentz-contracted volume during the early stage of the collision. The whole process of a collision is then considered as the expansion and the subsequent break-up into particles from an initial state. This approach resembles the 
Landau phenomenological hydrodynamic approach of multiparticle production in relativistic particle interactions [3], which was found to be in good agreement with the multiplicity data in particle and nuclear collisions in the wide energy range. In the picture proposed in $[15,16]$, the Landau hydrodynamics is combined with the constituent quark model [17]. This makes the secondary particle production to be basically driven by the amount of the initial effective energy deposited by participants-quarks or nucleons, into the Lorentz contracted overlap region. In $p p / \bar{p} p$ collisions, a single constituent (or dressed) quark from each nucleon takes part in a collision and rest are considered as spectators. Thus, the effective energy for the production of secondary particles is the energy carried by a single quark pair i.e. 1/3 of the entire nucleon energy. In contrary, in the head-on heavy-ion collisions, the participating nucleons are considered colliding by all three constituent quarks from each nucleon which makes the whole energy of the colliding nucleons (participants) available for secondary particle production. Thus, one expects that bulk observables measured in the head-on heavyion collisions at the c.m. energy per nucleon, $\sqrt{s_{N N}}$, to be similar to those from $p p / \bar{p} p$ collisions but at a three times larger c.m. energy i.e. $\sqrt{s_{p p}} \simeq 3 \sqrt{s_{N N}}$.

Combining the above-discussed ingredients of the constituent quarks and Landau hydrodynamics, one obtains the relationship between charged particle rapidity density per participant pair, $\rho(\eta)=\left(2 / N_{\text {part }}\right) \mathrm{d} N_{\mathrm{ch}} / \mathrm{d} \eta$ at midrapidity $(\eta \approx 0)$ in heavy-ion collisions and that in $p p / \bar{p} p$ collisions:

$$
\frac{\rho(0)}{\rho_{p p}(0)}=\frac{2 N_{\mathrm{ch}}}{N_{\mathrm{part}} N_{\mathrm{ch}}^{p p}} \sqrt{\frac{L_{p p}}{L_{N N}}} .
$$

In Eq. (1) the relation of the pseudorapidity density and the mean multiplicity is applied in its Gaussian form as obtained in Landau hydrodynamics. The factor $L$ is defined as $L=\ln (\sqrt{s} / 2 m)$. According to the approach considered, $m$ is the proton mass, $m_{p}$, in nucleus-nucleus collisions and the constituent quark mass in $p p / \bar{p} p$ collisions which is set to $\frac{1}{3} m_{p} . N_{\mathrm{ch}}$ and $N_{\mathrm{ch}}^{p p}$ are the mean multiplicities in nucleusnucleus and nucleon-nucleon collisions, respectively, and $N_{\text {part }}$ is the number of participants. Then one evolves Eq. (1) for the rapidity density $\rho(0)$ and the multiplicity $N_{\mathrm{ch}}$ at $\sqrt{s_{N N}}$, and the rapidity density $\rho_{p p}(0)$ and the multiplicity $N_{\mathrm{ch}}^{p p}$ at $3 \sqrt{s_{N N}}$ :

$$
\begin{aligned}
& \rho(0)=\rho_{p p}(0) \frac{2 N_{\mathrm{ch}}}{N_{\mathrm{part}} N_{\mathrm{ch}}^{p p}} \sqrt{1-\frac{4 \ln 3}{\ln \left(4 m_{p}^{2} / s_{N N}\right)}}, \\
& \sqrt{s_{N N}}=\sqrt{s_{p p}} / 3 .
\end{aligned}
$$

It was found $[15,16]$ that the current approach is able to reproduce very well the data on the c.m. energy dependence of the midrapidity density measured in the most central heavy-ion collisions by interrelating by Eq. (2) the measure- ments in hadronic and nuclear collisions up to the top RHIC energy. Moreover, it was also shown that similarly, the total multiplicities in these types of collisions follow the energydependence universality. Furthermore, the proposed factor $1 / 3$ allows one to relate both the multiplicity and midrapidity c.m. energy dependence in $e^{+} e^{-}$and $p p / \bar{p} p$ interactions and solves the problem of the factor $1 / 2$, the latter been introduced in [18] to account for the half of the energy lost attributed to the leading protons. If the factor $1 / 2$ is found to lead to some similarity in the multiplicity data, it cannot encompass the comparison of the midrapidity density. Interestingly, the 3NLO perturbative QCD [19-21] fit to $e^{+} e^{-}$ data has been shown [22] to describe the multiplicity measurements in $p p / \bar{p} p$ interactions up to $\mathrm{TeV}$ energies provided the inelasticity is set to $\approx 0.35$, i.e. the effective $1 / 3$ energy in hadronic interactions. Earlier, the factor $1 / 3$ has been already shown to provide an agreement in $e^{+} e^{-}$and $p p / \bar{p} p$ mean multiplicity data [23]; for a discussion, see [13]. Such a universality is found to correctly predict $[15,16]$ the value of the midrapidity density in $p p$ interactions at the $\mathrm{TeV}$ LHC energies [24].

In this paper, we extend the above-discussed approach of the constituent quark participants and Landau hydrodynamics to the midrapidity pseudorapidity-density dependence on the number of (nucleon) participants. Based on this energy dissipation picture, we apply effective-energy consideration to the pseudorapidity density of the transverse energy at midrapidity, namely to the dependence of this observable on the c.m. energy and on the number of participants measured in heavy-ion collisions in the RHIC and LHC experiments. We give predictions for foreseen higher-energy heavy-ion collisions at the LHC.

2. In Fig. 1, the charged particle pseudorapidity density per participant pair at midrapidity as a function of number of participants is shown as measured by PHOBOS experiment in AuAu collisions at RHIC at c.m. energy of $\sqrt{s_{N N}}=19.6$ to $200 \mathrm{GeV}$ [25] and by $\mathrm{CMS}$ experiment in $\mathrm{PbPb}$ collisions at LHC at $\sqrt{s_{N N}}=2.76 \mathrm{TeV}$ [26], respectively. The PHOBOS data at $\sqrt{s_{N N}}=200 \mathrm{GeV}$ multiplied by 2.12 are also shown to allow comparison with the LHC data and the current calculations. As it is noted above, this dependence cannot be reproduced by the wounded nucleon model where a number-of-nucleon-participant scaling is expected.

Within the above-discussed model of constituent quarks and Landau hydrodynamics, we consider this dependence in terms of centrality. The centrality is considered to characterize the degree of overlapping of the volumes of the two colliding nuclei, determined by the impact parameter. The most central collisions correspond therefore to the lowest centrality while the larger centrality defines more peripheral collisions. The centrality is closely related to the number of nucleon participants determined using a Monte Carlo 


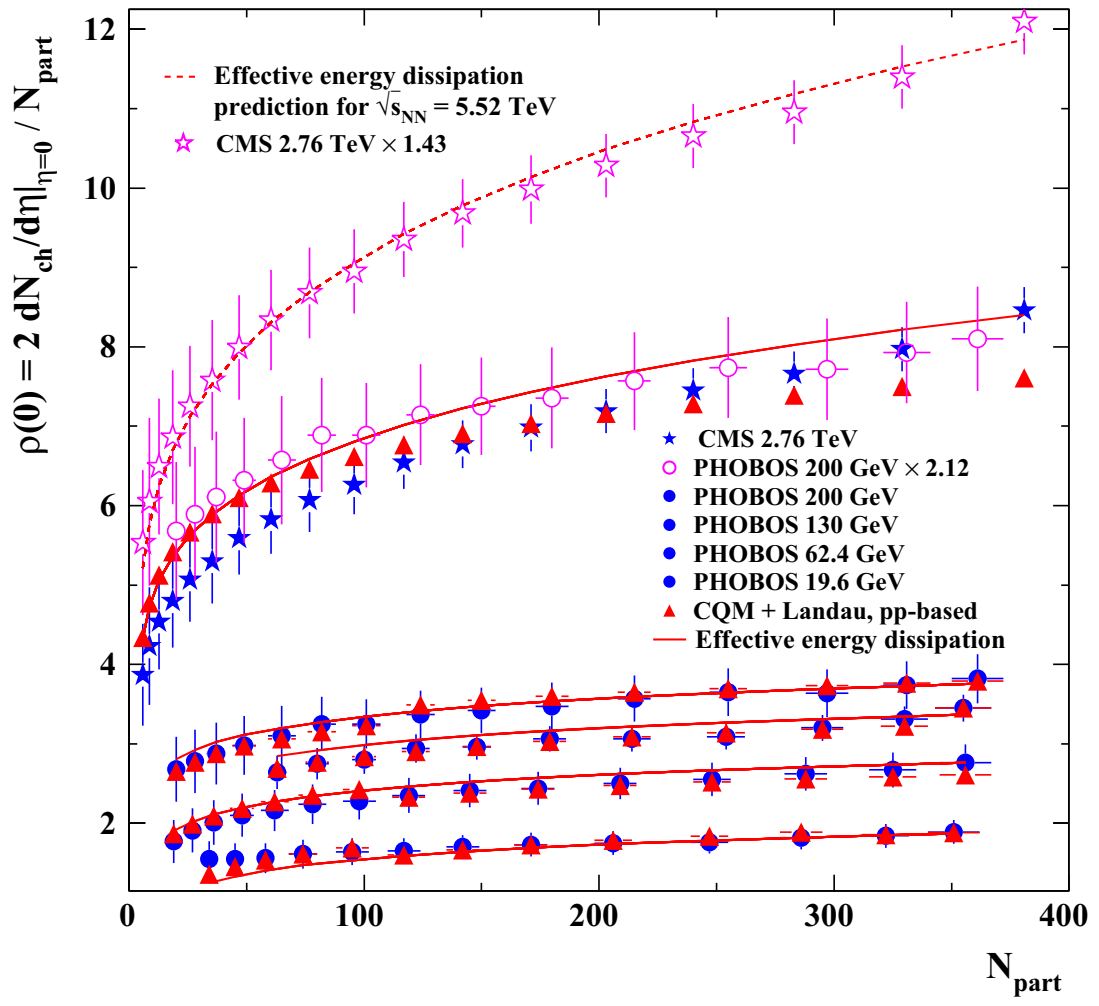

Fig. 1 The charged particle pseudorapidity density at midrapidity per participant pair as a function of the number of participants, $N_{\text {part }}$. The solid circles show the dependence measured in AuAu collisions at RHIC by PHOBOS at $\sqrt{s_{N N}}=19.6-200 \mathrm{GeV}$ [25] (bottom-top). The solid stars show the measurements in PbPb collisions at LHC by CMS at $\sqrt{s_{N N}}=2.76 \mathrm{TeV}$ [26]. The solid triangles show the calculations by

Glauber calculations so that the largest number of participants contribute to the most central heavy-ion collisions. Hence the centrality is related to the energy released in the collisions, i.e. the effective energy, $\varepsilon_{N N}$, which, in the framework of the proposed approach, can be defined as a fraction of the c.m. energy available in a collision according to the centrality, $\alpha$ :

$\varepsilon_{N N}=\sqrt{s_{N N}}(1-\alpha)$.

Conventionally, the data are divided into classes of centrality, or centrality intervals, so that $\alpha$ is the average centrality for the centrality interval, e.g. $\alpha=0.025$ for $0-5 \%$ centrality, which refers to the $5 \%$ most central collisions. In what follows we have checked that for a particular centrality interval the conclusions and the results are not influenced by taking either the mid-point of the centrality interval or both the extremes.

In fact, each of the scalings described by Eqs. (2) and (3) regulates a particular physics ingredient used in the modeling of our approach. Namely, the scaling introduced by Eq. (2) embeds the constituent quark model which leads to establishing a similarity between hadronic and nuclear collisions,
Eq. (4) using $p p / \bar{p} p$ data. The lines represent the effective-energy dissipation approach predictions based on the hybrid fit to the c.m. energy dependence of the midrapidity density in central heavy-ion collisions shown in Fig. 2. The open circles show the PHOBOS measurements at $\sqrt{s_{N N}}=200 \mathrm{GeV}$ multiplied by 2.12 , while the open stars show the CMS measurements multiplied by 1.43

while the scaling driven by Eq. (3) is appealed to define the energy budget effectively retained for multiparticle production in the most central collisions to determine the variables obtained from centrality data.

Then, for the effective c.m. energy $\varepsilon_{N N}$, Eq. (2) reads

$$
\begin{aligned}
\rho(0) & =\rho_{p p}(0) \frac{2 N_{\mathrm{ch}}}{N_{\mathrm{part}} N_{\mathrm{ch}}^{p p}} \sqrt{1-\frac{2 \ln 3}{\ln \left(2 m_{p} / \varepsilon_{N N}\right)}}, \\
\varepsilon_{N N} & =\sqrt{s_{p p}} / 3
\end{aligned}
$$

where $N_{\mathrm{ch}}$ is the mean multiplicity in central nucleusnucleus collisions measured at $\sqrt{s_{N N}}=\varepsilon_{N N}$. The rapidity density $\rho_{p p}(0)$ and the multiplicity $N_{\text {ch }}^{p p}$ are taken from the existing data or, where not available, calculated using the corresponding experimental c.m. energy fits ${ }^{1}$, and, according to the consideration, the calculations are made at $\sqrt{s_{p p}}=$ $3 \varepsilon_{N N}$. The $N_{\text {ch }}$ values are as well taken from the measurements in central heavy-ion collisions wherever available,

\footnotetext{
1 The E735 power-law fit $N_{\mathrm{ch}}^{p p}=3.102 s_{p p}^{0.178}$ [22] is used, while the linear-log fit $\rho_{p p}=-0.308+0.276 \ln \left(s_{p p}\right)$ [22] and the power-law fit by CMS [26], $\rho_{p p}=-0.402+s_{p p}^{0.101}$, are used for $\sqrt{s_{p p}} \leq 53 \mathrm{GeV}$ and for $\sqrt{s_{p p}}>53 \mathrm{GeV}$, respectively.
} 
while for the non-existing data the "hybrid" fit [41] combining the linear-logarithmic and power-law regularities is used. This fit is inspired by the measurements as well as by theoretical considerations. It is observed that the logarithmic fit well describes the heavy-ion multiplicity data up to the top RHIC energy $[15,16,27]$; however, as the collision energy increases above $1-2 \mathrm{TeV}$ at the LHC, the data clearly show a preference for the power-law behavior [26-28] in the multiplicity dependence on $\sqrt{s_{N N}}$. From the theoretical description point of view, such a c.m. energy dependence is expected [42] as soon as the logarithmic dependence is considered to characterize the fragmentation source(s) while the power-law behavior is believed to come from the gluongluon interactions.

In the framework of the model of constituent quarks combined with Landau hydrodynamics, we calculate the centrality dependence of the charged particle midrapidity density using Eq. (4) to reproduce the centrality data shown in Fig. 1. The calculations are shown by solid triangles. One can see that within this approach where the collisions are derived by the centrality-defined effective c.m. energy $\varepsilon_{N N}$, the calculations are in very good overall agreement with the measurements independent of the collision energy. Similar results are obtained as the $N_{\text {part }}$-dependence of the PHENIX [30,31], STAR [32], or CuCu PHOBOS [25] measurements from RHIC and ALICE [40] or ATLAS [27] data from LHC are used (not shown). Some slightly lower values are, however, seen in the calculations compared to the data for some low- $N_{\text {part }}$, i.e. for the most peripheral collisions, at $\sqrt{s_{N N}}=19.6 \mathrm{GeV}$, and for a couple of central data points obtained at the highest $\sqrt{s_{N N}}$. The deviation observed in the peripheral collisions at $\sqrt{s_{N N}}=19.6 \mathrm{GeV}$ looks to be due to the experimental limitations and the extrapolation used in the reconstruction for the measurements in this region of very low multiplicity [25]. This also may explain the $N_{\text {part }}{ }^{-}$ scaling of the data at $\sqrt{s_{N N}}=19.6 \mathrm{GeV}$ in the most peripheral region so the data of these centrality intervals do not follow the common trend of decreasing as it is observed in higher-energy measurements. The low values obtained within the approach for a few most central collisions at the LHC energy can be explained by no data on $N_{\mathrm{ch}}^{p p}$ being available at $\sqrt{s_{p p}}>1.8 \mathrm{TeV}$. Moreover, for $\sqrt{s_{p p}}>53 \mathrm{GeV}$, the second-order logarithmic polynomial fit to the $\sqrt{s_{p p}}$ dependence of $N_{\mathrm{ch}}^{p p}$ is indistinguishable from the exponential function fit [22]. The latter regularity is used here for the $\sqrt{s_{p p}}$ dependence to calculate $N_{\mathrm{ch}}^{p p}$ above the Tevatron energy.

Given the obtained agreement between data and the calculations and considering the similarity put forward for $\varepsilon_{N N}$ and $\sqrt{s_{N N}}$, one would expect the measured centrality data at $\varepsilon_{N N}$ to follow the $\sqrt{s_{N N}}$ dependence of the midrapidity density in the most central nuclear collisions. In Fig. 2, the measurements of the charged particle pseudorapidity density at midrapidity in head-on nuclear collisions are plotted against the $\sqrt{s_{N N}}$ from a few $\mathrm{GeV}$ at GSI to a few $\mathrm{TeV}$ at the LHC along with the centrality data, shown as a function of $\varepsilon_{N N}$, from low-energy RHIC data by STAR at $9.2 \mathrm{GeV}$ [33], and the measurements, shown in Fig. 1, by PHOBOS [25] and CMS [26] experiments as a function of $\varepsilon_{N N}$. The centrality data effective-energy dependence follow well the data on the most central collision c.m. energy behavior.

We fit the weighted combination of the midrapidity density from the head-on collisions by the hybrid fit function

$$
\begin{aligned}
\rho(0)= & (-0.306 \pm 0.027)+(0.364 \pm 0.009) \ln \left(s_{N N}\right) \\
& +(0.0011 \pm 0.0011) s_{N N}^{(0.50 \pm 0.06)},
\end{aligned}
$$

which is, as it is noticed above, inspired by the measurements and supported by theoretical consideration. The fit combines the linear-log dependence on $\sqrt{s_{N N}}$ observed up to the top RHIC energy [25,30,31] and the power-law dependence obtained with the LHC data [26-28]. This fit is shown in Fig. 2 by the dashed line. One can see that the fit is as well close to the centrality data. To clarify, the weighted combination of the centrality data are also fitted by the hybrid function,

$$
\begin{aligned}
\rho(0)= & (0.002 \pm 0.080)+(0.646 \pm 0.022) \ln \left(\varepsilon_{N N}\right) \\
& +(0.0003 \pm 0.0001) \varepsilon_{N N}^{(1.158 \pm 0.034)}
\end{aligned}
$$

where, in addition to the low-energy STAR data and the measurements, shown in Fig. 1, by the PHOBOS and CMS experiments, the midrapidity-density data on the centrality dependence from ALICE [40], ATLAS [27], PHENIX [30] and STAR [32] are included (not shown). The fit is shown by the solid line and is very close to the fit made to the centrality data. From this one can conclude that the picture proposed well reproduces the data under the assumption of the effective energy deriving the multiparticle production process and pointing to the similarity in all the data from peripheral to the most central measurements to follow the same energy behavior. From the fit, we estimate the midrapidity-density value to be of about 12.0 within $10 \%$ uncertainty in the most central collisions at $\sqrt{s_{N N}}=5.52 \mathrm{TeV}$ shown by the solid circle in Fig. 2.

In addition to the hybrid fits, in Fig. 2 we show the linear$\log$ fit $[15,16]$ up to the top RHIC energy (thin dashed line) and the power-law fit for the entire energy range (dasheddotted line) to the most central collision data along with the power-law fit to the centrality data (dotted line). All the fits are made by using the weighted data as above. One can see that the power-law fit describes well the head-on collision measurements (see also [14]) and, within the errors, does not differ from the linear-log or the hybrid functions up to the RHIC energies. However, it deviates from the most central collision hybrid fit as soon as the LHC measurements are included. The power-law fit to the centrality data are much closer to the hybrid fits, and it is almost indistinguish- 


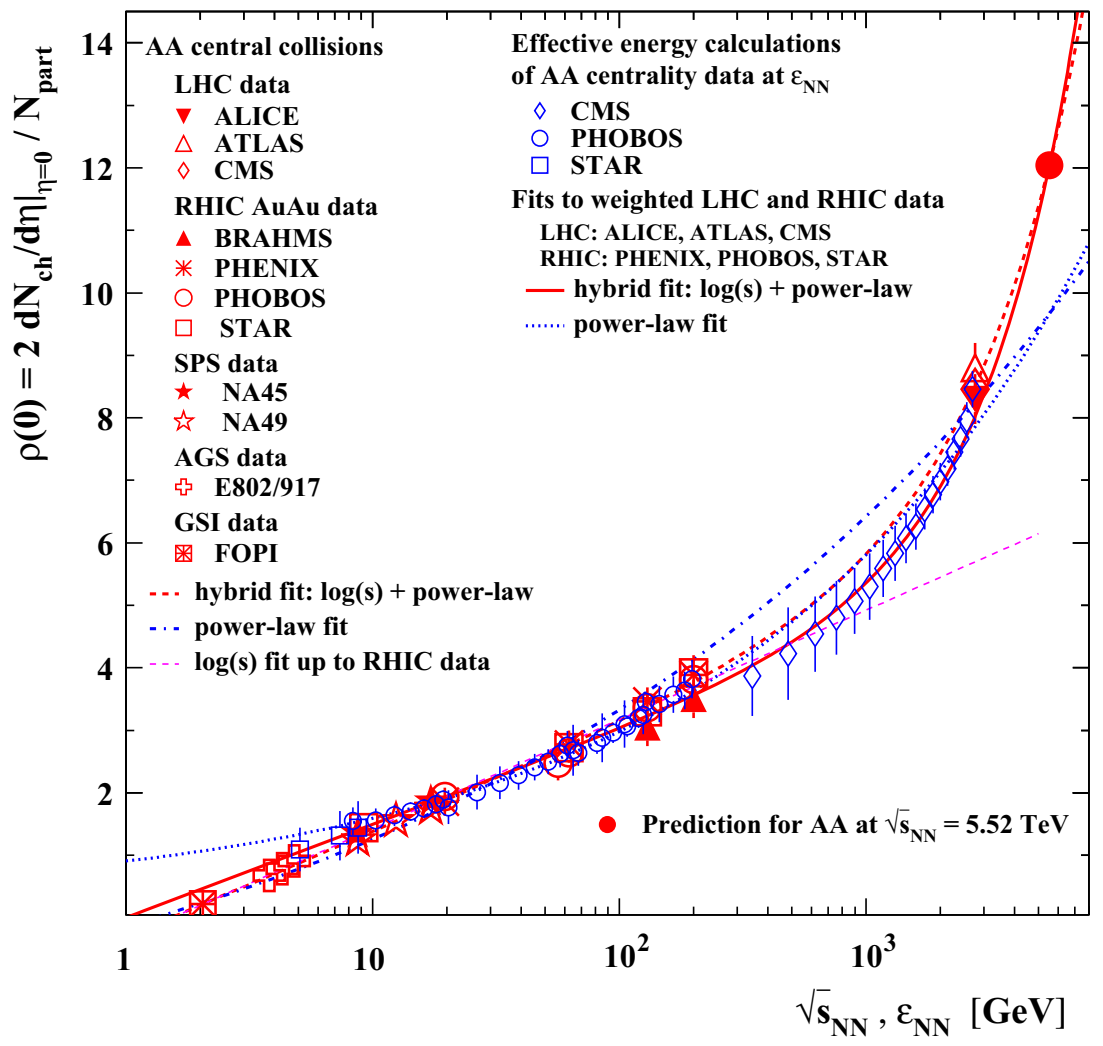

Fig. 2 The charged particle pseudorapidity density per participant pair at midrapidity as a function of c.m. energy per nucleon, $\sqrt{s_{N N}}$, in central nucleus-nucleus (AA) collisions (shown by large symbols), and as a function of effective c.m. energy, $\varepsilon_{N N}$ [Eq. (3)], for AA collisions at different centrality (small symbols). The data of central AA collisions are from: the $\mathrm{PbPb}$ measurements at LHC by ALICE [28], ATLAS [27], and CMS [26] experiments; the AuAu measurements at RHIC by BRAHMS [29], PHENIX [30,31], PHOBOS [25], and STAR [32,33] experiments; the values recalculated in [30] from the measurements at CERN SPS by CERES/NA45 [34] and NA49 [35,36] experiments, at Fermilab AGS by E802 [37] and E917 [38] experiments, and at GSI by FOPI Collab. [39] (estimated in [30]). The centrality data represent the measurements by CMS [26], PHOBOS [25], and STAR [33]; the CMS and PHOBOS data are those from Fig. 1, while for clarity,

able from the hybrid fit to the central data up to the head-on collision LHC points. Both the power-law fits, to the headon collision data and to the centrality data, give predictions close to each other but lower than the hybrid fits up to some higher c.m. energies. Interestingly, using the approach of the effective-energy dissipation, one can clearly see the transition to a possibly new regime in the multihadron production in heavy-ion collisions demonstrated by the data as $\sqrt{s_{N N}}$ increases up to about $600-700 \mathrm{GeV}$ per nucleon. The centrality data still follow the central collision data and the log fit up to these energies while then the energy behavior changes to the power-law one. The change in the $\sqrt{s_{N N}}$-dependence from the logarithmic to the power-law one seems to be a reason of lower-value predictions by theoretical models [28]. The change also restrains predictions for heavy-ions within the universality picture $[15,16]$ which, however, gives the just every second point of the PHOBOS measurements is shown. The dashed-dotted line and the dashed line show the fits to the central collision data: the power-law fit, $\rho(0)=-2.955+2.823 s_{N N}^{0.087}$, and the hybrid fit, $\rho(0)=-0.306+0.364 \ln \left(s_{N N}\right)+0.0011 s_{N N}^{0.5}$. The thin dashed line shows the linear-log fit, $\rho(0)=-0.327+0.381 \ln \left(s_{N N}\right)$ $[15,16]$ to the central collision data up to the top RHIC energy. The dotted line and the solid line show the fits to the centrality data: the power-law fit, $\rho(0)=0.244+0.663 \varepsilon_{N N}^{0.308}$, and the hybrid fit, $\rho(0)=0.002+0.646 \ln \left(\varepsilon_{N N}\right)+0.0003 \varepsilon_{N N}^{1.158}$, respectively. The fitted centrality data include, except of the shown data, also the measurements by ALICE [40] and ATLAS [27] at the LHC, and by PHENIX [30] and STAR [32,33] at RHIC (not shown). The solid circle shows the prediction for $\sqrt{s_{N N}}=5.52 \mathrm{TeV}$

correct predictions for $p p / \bar{p} p$ [24], where both the logarithmic [43] and the power-law [26] functions provide equally good fits to the data up to $\sqrt{s_{p p}}=7 \mathrm{TeV}$.

Now, using the effective c.m. energy approach, we apply the obtained hybrid function fit of the midrapidity density measured in head-on collision data, Eq. (5), to the centrality data, shown in Fig. 1 as a function of $N_{\text {part }}$. The calculations are shown by the solid lines. One can see that the approach well describes the measurements and actually follows the predictions by Eq. (4), except the LHC data, where it is better than the calculations of Eq. (4), though slightly overshoots the measurements. Similar to the consideration combining constituent quarks and Landau hydrodynamics, the calculations using the effective energy $\varepsilon_{N N}$ show lower values for the very peripheral points at the lowest c.m. energy, $\sqrt{s_{N N}}=19.6 \mathrm{GeV}$. The difference, as mentioned 
above, seems to be due to the difficulties in the measurements because of the very low multiplicity in these data. A slight overestimation of the LHC data is due to the fact that the fit of Eq. (5) uses the highest (0-2\% centrality) ATLAS point of the head-on collisions.

Similarly to the above calculations for the existing data on the $N_{\text {part }}$-dependence of the midrapidity density, we made the predictions for the forthcoming heavy-ion collisions at $\sqrt{s_{N N}}=5.52 \mathrm{TeV}$. The predictions are shown by the dashed line in Fig. 1, where the centrality and $N_{\text {part }}$ values are taken as in the $2.76 \mathrm{TeV}$ data shown. The expectations show increase of the $\rho(0)$ with $N_{\text {part }}$ (decrease with centrality) from about 5 to 12 . The increase looks to be faster than at $\sqrt{s_{N N}}=2.76 \mathrm{TeV}$, especially for the peripheral region, similar to the change in the behavior seen as one moves from the RHIC measurements to the LHC data, cf. $200 \mathrm{GeV}$ data and 2.76 TeV data in Fig. 2. We find that the predictions made here are well reproduced when the LHC data are scaled by a factor 1.43, similar to the multiplication factor (of 2.12 shown here) found $[26,27,40]$ to reproduce the $2.76 \mathrm{TeV}$ LHC data by the $200 \mathrm{GeV}$ RHIC ones.

Interestingly, within the picture of the effective-energy dissipation of constituent quark participants one can explain the observed similarity of the midrapidity densities measured in $p p / \bar{p} p$ interactions and in heavy-ion collisions at the same c.m. energy, as soon as in the latter case the data are recalculated in the constituent quark framework [8-11]. Moreover, this approach supports the scaling with the number of partcipants of the midrapidity pseudorapidity and transverse energy densities obtained for RHIC [6-11,44] and LHC [41] data in the constituent quark framework. Note that this scaling been observed also for most peripheral collisions may be understood in the framework of the approach proposed here by considering the most peripheral collisions to be driven by nucleon-nucleon interactions where a pair of participating constituent quarks each per nucleon contribute, thus the fraction of c.m. energy, i.e. the effective energy of the participants is pumped into the small collision zone of the overlapped nuclei.

3. The effective c.m. energy approach applied to the charged particle pseudorapidity density at midrapidity can be considered to be applied to another important variable, such as the pseudorapidity density of the transverse energy, $\rho_{T}(\eta)=$ (2/ $\left.N_{\text {part }}\right) \mathrm{d} E_{T} / \mathrm{d} \eta$, at midrapidity, $\eta \approx 0$. The charged particle density and the transverse energy density are closely related and, been studied together, provide important characteristics of the underlying dynamics of the multihadron production. The transverse energy measurements, as well as the pseudorapidity data, have been shown to be reasonably well modeled by the constituent quark picture $[7,41,44]$.

In Fig. 3, the $\sqrt{s_{N N}}$ dependence of the charged particle midrapidity transverse energy density in pseudorapidity is displayed as measured in head-on collisions at the experiments from a few GeV at GSI to a few TeV at the LHC, shown by the big symbols. On top of these data the centrality data from the PHENIX experiment at RHIC [44] and the CMS experiment at LHC [45] are added as a function of the effective c.m. energy $\varepsilon_{N N}$ shown by small symbols. Similarly to the case of the charged particle density at midrapidity, the $E_{T}$ density data show the complementarity of these two types of measurements: the centrality data follow well the data from the central collisions.

To better trace the similarity in the energy dependence of the central collision and the centrality-dependent data, we fit the data by the hybrid function, as is done in Fig. 2 for the particle psudorapidity densities. For the central collisions one gets

$$
\begin{aligned}
\rho_{T}(0)= & (-0.447 \pm 0.014)+(0.327 \pm 0.011) \ln \left(s_{N N}\right) \\
& +(0.002 \pm 0.003) s_{N N}^{0.50 \pm 0.08}
\end{aligned}
$$

and similar fit to the centrality data reads

$$
\begin{aligned}
\rho_{T}(0)= & (-0.387 \pm 0.090)+(0.574 \pm 0.032) \ln \left(\varepsilon_{N N}\right) \\
& +(0.011 \pm 0.005) \varepsilon_{N N}^{0.818 \pm 0.064} .
\end{aligned}
$$

The fits are shown in Fig. 3 by the dashed and solid lines, respectively. The data from different experiments are weighted, and the fit of the effective c.m. energy $\varepsilon_{N N}$ includes the STAR measurements in addition to the PHENIX ones. One can see that the two fits are amazingly close to each other for the entire energy range allowing to conclude that the effective-energy approach provides a good description of the $E_{T}$ production in heavy-ion collisions. We estimate the value of $\rho_{T}(0)$ to be about $16.9 \mathrm{GeV}$ with about $10 \%$ uncertainty for most central collisions at $\sqrt{s_{N N}}=5.52 \mathrm{TeV}$ shown by the solid circle in Fig. 3 .

As is obtained above for the midrapidity pseudorapiditydensity energy dependence (Fig. 2), in Fig. 3 the LHC data demonstrate a clear departure from the linear-log regularity in the region of $\sqrt{s_{N N}} \simeq 500-700 \mathrm{GeV}$; the $\log$ fit to the data up to the top RHIC energy is shown by the thin dashed line and is taken from [30]. This observation supports a possible transition to a new regime in heavy-ion collisions at $\sqrt{s_{N N}}$ above a few hundred $\mathrm{GeV}$ as indicated by the midrapidity density in Fig. 1. In Fig. 3, we also show the power-law fits to the central collision measurements by the dashed-dotted line and to the centrality data by the dotted line. As above, in the fits the data from different experiments are weighted. One can see that the power-law fit to the central collision data underestimates the LHC measurement at $2.76 \mathrm{TeV}$ data and deviates from the data at $\sqrt{s_{N N}} \sim 1 \mathrm{TeV}$. However, the power-law fit to the centrality describes well the data in the full available c.m.-energy region, though lies slightly lower than the hybrid fit, Eq. (8). Meantime, this fit overestimates the data below $\sqrt{s_{N N}} \approx 10 \mathrm{GeV}$, similarly to the case of 


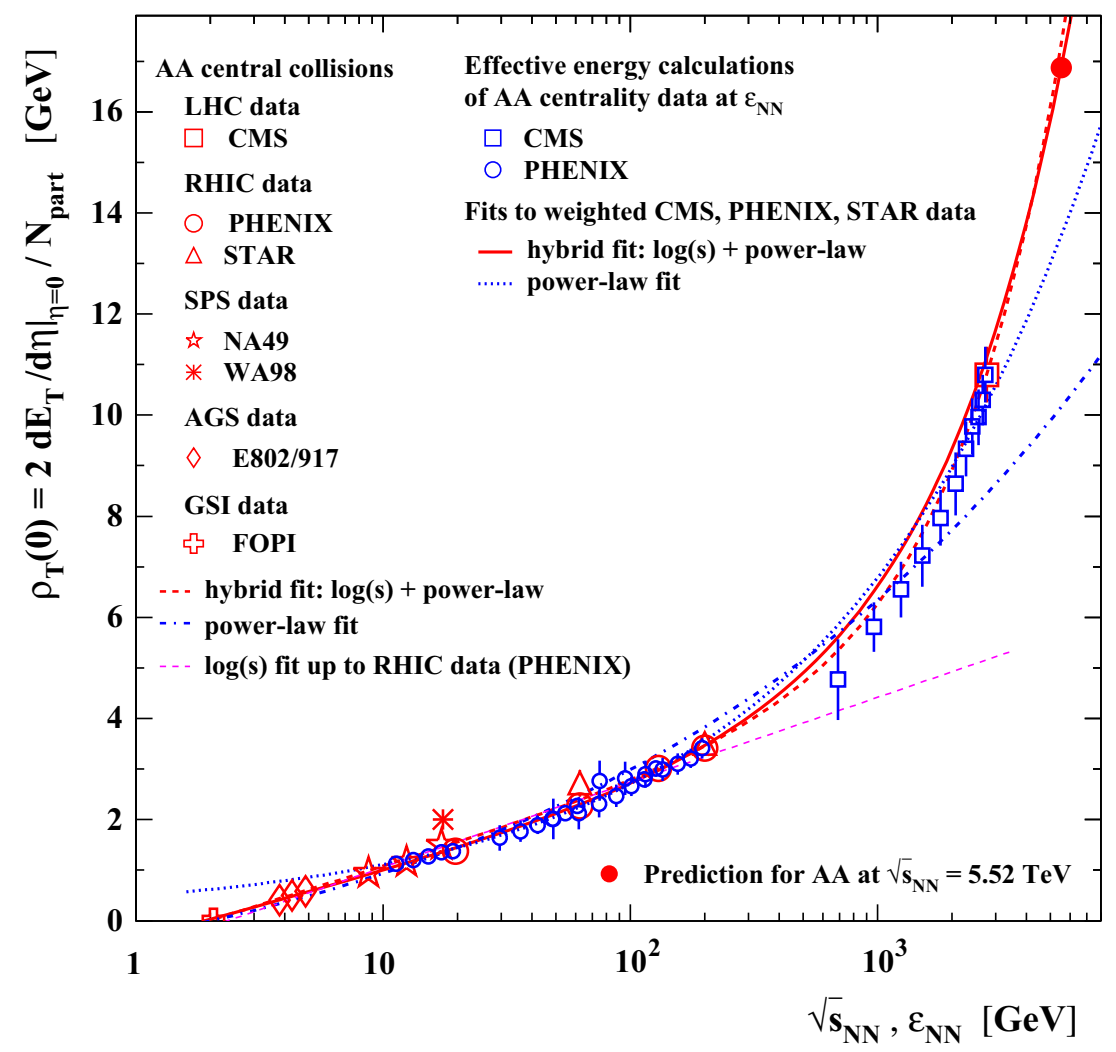

Fig. 3 The charged particle transverse energy pseudorapidity density per participant pair at midrapidity as a function of c.m. energy per nucleon, $\sqrt{s_{N N}}$, in central nucleus-nucleus (AA) collisions (shown by large symbols), and as a function of effective energy, $\varepsilon_{N N}$ [Eq. (3)], for AA collisions at different centrality (small symbols). The data of central AA collisions are from: the $\mathrm{PbPb}$ measurements at LHC by CMS [45] experiment; the AuAu measurements at RHIC by PHENIX $[30,44]$ and STAR $[46,47]$ experiments; the values recalculated in [30] from the measurements at CERN SPS by CERES/NA45 [35,36] and WA98 [48] experiments, at Fermilab AGS by E802 and E917 experiments [49], and at GSI by FOPI Collab. [39] (estimated in [30]). The centrality data represent the measurements by CMS at the LHC [45] and by PHENIX at RHIC [30,44]; the CMS and PHENIX data

the multiplicity data on centrality, Fig. 2. Interestingly, the shown power-law fit curve to the centrality data is similar to that obtained by CMS for $\sqrt{s_{N N}} \geq 8.7 \mathrm{GeV}$ [45]; moreover, fitting all the $E_{T}$ centrality data à la CMS, one finds a good fit to the data by $\rho_{T}(0)=0.43 \varepsilon_{N N}^{0.20}$ (not shown) which resembles the CMS fit, $\rho_{T}(0)=0.46 s_{N N}^{0.20}$, to the head-on collision data. This again demonstrates the multihadron production in heavy-ion collisions to be well described by the effective c.m. energy dissipation picture.

To further exploit the effective-energy approach with the centrality data, in Fig. 4 we show the $N_{\text {part }}$ dependence of the centrality data from Fig. 3 along with the central collision data fit, Eq. (7), but as a function of the centralitydependent c.m. effective energy $\varepsilon_{N N}$. One can see that the fit well describes the data; in this case the agreement is even better than for the midrapidity density, as one concludes from the comparison with the LHC centrality data. Inter- are those from Fig. 4, while for clarity, just every second point of the PHENIX measurements is shown. The dashed-dotted line and the dashed line show the fits to the central collision data: the powerlaw fit, $\rho_{T}(0)=-2.29+1.97 s_{N N}^{0.107}$, and the hybrid fit, $\rho_{T}(0)=$ $-0.447+0.327 \ln \left(s_{N N}\right)+0.002 s_{N N}^{0.5}$. The thin dashed line shows the linear-log PHENIX fit [30] to the central collision data up to the top RHIC energy. The dotted line and the solid line show the fits to the centrality data: the power-law fit, $\rho_{T}(0)=0.09+0.40 \varepsilon_{N N}^{0.40}$, and the hybrid fit, $\rho_{T}(0)=-0.387+0.574 \ln \left(\varepsilon_{N N}\right)+0.011 \varepsilon_{N N}^{0.818}$, respectively. The fitted centrality data include, except of the shown data, also the measurements by STAR $[46,47]$ at RHIC (not shown). The solid circle shows the prediction for $\sqrt{s_{N N}}=5.52 \mathrm{TeV}$

estingly, the open circles which represent the RHIC data at $\sqrt{s_{N N}}=200 \mathrm{GeV}$ scaled by 3.07 , to allow comparison with the LHC measurements, demonstrate much less decrease as the centrality increases (more peripheral data), than that observed for the LHC data. This is different for the pseudorapidity density of charged particles at midrapidity measurements (see Fig. 2). In contrast to the scaled RHIC data, the effective-energy approach follows well the LHC measurements.

Similarly to the above comparison to the existing data on the $N_{\text {part }}$-dependence of the midrapidity transverse energy density, we make the predictions for the future heavy-ion collisions at $\sqrt{s_{N N}}=5.52 \mathrm{TeV}$ within the effective-energy dissipation approach. The predictions are shown by the dashed line in Fig. 3. The predictions show more rapid increase of the $\rho_{T}(0)$ with $N_{\text {part }}$ (decrease with centrality) than at $\sqrt{s_{N N}}=2.76 \mathrm{TeV}$, especially for the peripheral region, sim- 


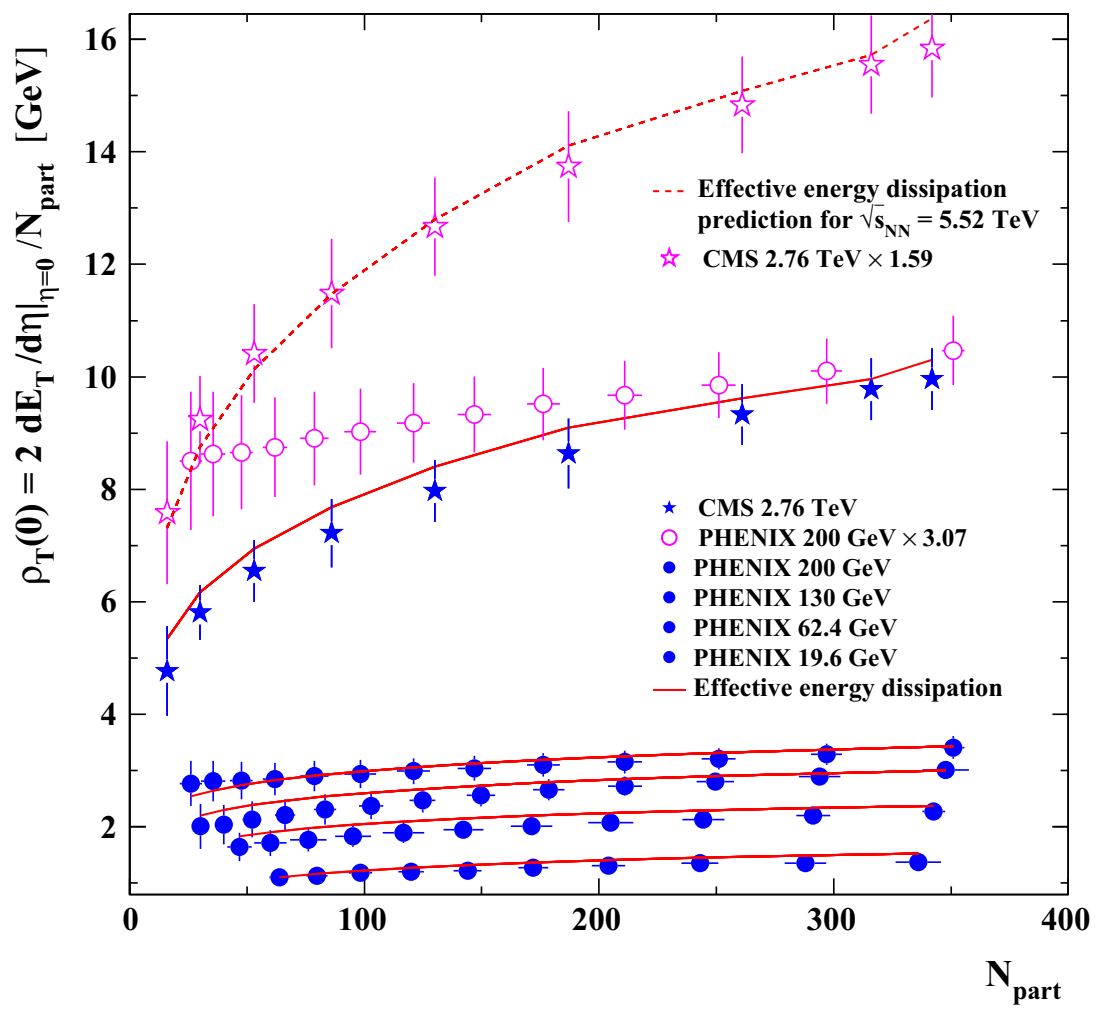

Fig. 4 The charged particle transverse energy pseudorapidity density at midrapidity per participant pair as a function of the number of participants, $N_{\text {part }}$. The solid symbols show the data from AuAu collisions at RHIC (circles) by PHENIX experiment at $\sqrt{s_{N N}}=19.6$ [30] and 62.4, 130 and $200 \mathrm{GeV}$ [44] (bottom-top) and from $\mathrm{PbPb}$ collisions at LHC by CMS at $\sqrt{s_{N N}}=2.76 \mathrm{TeV}$ [45] (stars). The lines show the

ilar to the change in the behavior seen as one moves from the RHIC measurements to the LHC data and similar to that obtained for the midrapidity density, Fig. 2. We find that the predictions made are well reproduced as the LHC data are scaled by a numerical factor 1.59 , as shown by open stars, Fig. 4.

4. In summary, we analyzed the midrapidity pseudorapidity density of charged particles and of the transverse energy measured in nucleus-nucleus collisions in the whole available range of the collision c.m. energy per nucleon, $\sqrt{s_{N N}}$, from a few $\mathrm{GeV}$ at GSI up to a few $\mathrm{TeV}$ at the LHC. The dependencies of these key variables on the c.m. energy per nucleon and on the number of participants (or centrality) have been revealed within the approach of the dissipation of the effective energy pumped in by the participants of the collisions, which forms the effective-energy budget in the multiparticle production process. Namely, the model of constituent quarks combined with Landau hydrodynamics is applied to reproduce the midrapidity-density dependence on the number of participants. This approach, proposed earlier in $[15,16]$ and pointed to the universality of the multihadron production in different types of collisions up to the top RHIC energy allows one to well predict the LHC measurements in predictions by the effective-energy approach using the hybrid fit to the c.m. energy dependence of the midrapidity transverse energy density in central heavy-ion collisions shown in Fig. 3. The open circles show the PHENIX measurements at $\sqrt{s_{N N}}=200 \mathrm{GeV}$ multiplied by 3.07, while the open stars show the CMS data multiplied by 1.59

$p p / \bar{p} p$ interactions on the midrapidity density of charged particles. Within this picture, we find that the dependence of the pseudorapidity density at midrapidity from the RHIC to LHC data is well reproduced as soon as the effective c.m. energy variable is introduced as the centrality-defined fraction of the collision c.m. energy. Based on this finding, it is shown that the most central collision data and the centralitydependent data follow a similar $\sqrt{s_{N N}}$ dependence obtained for the central collision data as soon as the centrality data is rescaled to the effective energy. The hybrid fit, combining the linear-log and the power-law c.m. energy dependencies of the head-on collision data, where the linear-log function known to fit the measurements up to the top RHIC energy and the power-law regularity is needed up to the $\mathrm{TeV}$ LHC data, is found to well reproduce the dependence of the midrapidity densities on the number of participants within the effective-energy approach. Similar observations are made for the transverse energy midrapidity-density measurements: as soon as the centrality data is recalculated for the c.m. effective energy, these measurements are found to well complement the central collision data c.m. energy behavior. The hybrid fit made to the central collision data is shown to reproduce well the midrapidity transverse energy dependence on 
the number of participants. For both the variables studied, a clear departure of the data as a function of the effective c.m. energy from the linear-log dependence to the power-law one is observed at $\sqrt{s_{N N}} \simeq 500-700 \mathrm{GeV}$ indicating a possible transition to a new regime in heavy-ion collisions. The data at $\sqrt{s_{N N}} \sim 1 \mathrm{TeV}$ would be extremely useful to clarify the observations made here. Based on the hybrid fits in the framework of the discussed approach, the predictions for the energy and the number-of-participant dependencies for the measurements in the forthcoming heavy-ion runs at LHC at $\sqrt{s_{N N}}=5.52 \mathrm{TeV}$ are made.

Open Access This article is distributed under the terms of the Creative Commons Attribution License which permits any use, distribution, and reproduction in any medium, provided the original author(s) and the source are credited.

Funded by $\mathrm{SCOAP}^{3}$ / License Version CC BY 4.0.

\section{References}

1. W. Heisenberg, Zs. Phis. 126, 569 (1949)

2. E. Fermi, Prog. Theor. Phys. 5, 570 (1950)

3. L.D. Landau, Izv. Akad. Nauk. Ser. Fiz. 17, 51 (1953). English translation: Collected Papers of L.D. Landau, ed. by D. Ter-Haarp (Pergamon, Oxford, 1965), p. 569. Reprinted in: Quark-Gluon Plasma: Theoretical Foundations, ed. by J. Kapusta, B.Müller, J. Rafelski (Elsevier, Amsterdam, 2003), p. 283

4. A. Białas, B. Bleszyński, W. Czyż, Nucl. Phys. B 111, 461 (1976)

5. A. Białas, J. Phys. G 35, 044053 (2008)

6. S. Eremin, S. Voloshin, Phys. Rev. C 67, 064905 (2003)

7. P.K. Netrakanti, B. Mohanty, Phys. Rev. C 70, 027901 (2004)

8. B. De, S. Bhattacharyya, Phys. Rev. C 71, 024903 (2005)

9. R. Nouicer, AIP Conf. Proc. 828, 11 (2006)

10. R. Nouicer, AIP Conf. Proc. 842, 86 (2006)

11. R. Nouicer, Eur. Phys. J. C 49, 281 (2007)

12. PHOBOS Collab., B.B. Back et al., Phys. Rev. C 74, 021902 (2006). nucl-ex/0301017

13. W. Kittel, E.A. De Wolf, Soft Multihadron Dynamics (World Scientific, Singapore, 2005)

14. R. Singh, L. Kumar, P.K. Netrakanti, B. Mohanty, Adv. High Energy Phys. 2013, 761474 (2013)
15. E.K.G. Sarkisyan, A.S. Sakharov, Eur. Phys. J. C 70, 533 (2010)

16. E.K.G. Sarkisyan, A.S. Sakharov, AIP Conf. Proc. 828, 35 (2006)

17. V.V. Anisovich, N.M. Kobrinsky, J. Nyiri, Yu.M. Shabelsky, Quark Model and High Energy Collisions (World Scientific, Singapore, 2004)

18. PHOBOS Collab., B.B. Back et al., Nucl. Phys. A 757, 28 (2005)

19. I.M. Dremin, J.W. Gary, Phys. Lett. B 459, 341 (1999)

20. A. Capella et al., Phys. Rev. D 61, 074009 (2000)

21. I.M. Dremin, J.W. Gary, Phys. Rep. 349, 301 (2001)

22. J.F. Grosse-Oetringhaus, K. Reygers, J. Phys. G 37, 083001 (2010)

23. P.V. Chliapnikov, V.A. Uvarov, Phys. Lett. B 251, 192 (1990)

24. R. Rougny (for the CMS Collab.), Nucl. Phys. B (Proc. Suppl.) 207-208, 29 (2010)

25. B. Alver et al., Phys. Rev. C 83, 024913 (2011)

26. CMS Collab., S. Chatrchyan et al., J. High Energy Phys. 08, 141 (2011)

27. ATLAS Collab., G. Aad et al., Phys. Lett. B 710, 363 (2012)

28. ALICE Collab., K. Aamodt et al., Phys. Rev. Lett. 105, 252301 (2010)

29. BRAHMS Collab., I.G. Bearden et al., Phys. Rev. Lett. 94, 162301 (2005)

30. PHENIX Collab., S.S. Adler et al., Phys. Rev. C 74, 049901 (2005). ibid. 71, 034908 (E) (2005)

31. A. Milov (for the PHENIX Collab.), J. Phys. Conf. Ser. 5, 17 (2005)

32. STAR Collab., B.I. Abelev et al., Phys. Rev. C 79, 034909 (2009)

33. STAR Collab., B.I. Abelev et al., Phys. Rev. C 81, 024911 (2010)

34. F. Ceretto (for the CERES/NA45 Collab.), Nucl. Phys. A 638, 467c (1998)

35. F. Siklér (for the NA49 Collab.), Nucl. Phys. A 661, 45c (1999)

36. NA49 Collab., S.V. Afanasiev et al., Phys. Rev. C 66, 054902 (2002)

37. E802 Collab., L. Ahle et al., Phys. Rev. C 59, 2173 (1999)

38. E917 Collab., B.B. Back et al., Phys. Rev. Lett. 86, 1970 (2001)

39. FOPI Collab., W. Reisdorf et al., Nucl. Phys. A 612, 493 (1997)

40. ALICE Collab., K. Aamodt et al., Phys. Rev. Lett. 106, 032301 (2011)

41. R. Sahoo, A.N. Mishra, Int. J. Mod. Phys. E 23, 1450024 (2014)

42. G. Wolschin, J. Phys. G 40, 045104 (2013)

43. CMS Collab., V. Khachatryan et al., Phys. Rev. Lett. 105, 022002 (2010)

44. PHENIX Collab., S.S. Adler et al., Phys. Rev. C 89, 044905 (2014)

45. CMS Collab., S. Chatrchyan et al., Phys. Rev. Lett. 109, 152303 (2012)

46. J. Adams et al., Phys. Rev. C 70, 054907 (2004)

47. R. Sahoo, Ph.D. thesis (Utkal University, 2007), arXiv:0804.1800

48. WA98 Collab., M.M. Aggarval et al., Eur. Phys. J. C 18, 651 (2001)

49. E802 Collab., T. Abbott et al., Phys. Rev. C 63, 064602 (2001) 